\title{
Provision of dental services at a single institution in the UK's epicentre during the COVID-19 pandemic
}

\author{
Sanford Grossman, ${ }^{1}$ Pradeep Sandhu, ${ }^{1}$ Christopher Sproat ${ }^{1}$ and Vinod Patel ${ }^{\star 1}$
}

\section{Key points}

Access to urgent dental care was limited in the earlier phase of the UK's COVID-19 lockdown and cessation of dental practice limited face-to-face consultations
Remote triage with the use of advice, analgesia and antimicrobials provides limited relief for patients with diagnoses of necrotic pulp nature.
Personal protective equipment categorised based upon the intervention being delivered allowed for continual delivery of urgent dental services.

\begin{abstract}
Coronavirus disease 2019 (COVID-19) has had a significant impact on dentistry in the UK due to the perceived risks associated with infection control. These concerns have led to a complete paralysis of routine dental care with provisions for emergency dental care only, which have been scarce. Considering the latter, this article presents a service evaluation of a hospital-based acute dental care service, which was rapidly adapted and enhanced to continue managing dental emergencies during the COVID-19 pandemic. The analysis is based upon the dental diagnoses, management and geography of travel of over 1,500 attending patients in a five-week period during the rise and peak of COVID-19 in the UK. In addition, we assess our dental workforce's COVID-19 sickness reporting for those providing urgent dental care within this enhanced service. The article aims to provide additional and valuable frontline clinical information, experience and outcomes, including our categorisation of personal protective equipment used for varying face-to-face dental management during COVID-19.
\end{abstract}

\section{Introduction}

The emergence of a novel coronavirus (SARSCoV-2) commencing in Wuhan, China, in December $2019^{1}$ has subsequently led to a global outbreak, prompting the World Health Organisation (WHO) to declare a pandemic. ${ }^{2}$ The continual and rapid international rise $^{3}$ has been labelled a major and unprecedented challenge to public healthcare and government systems.

In the UK, the first half of March 2020 saw the first 3,000 cases and 50 deaths from COVID-19, ${ }^{4}$ resulting in radical government action on 23 March 2020. ${ }^{5}$ Several mandates such as a national 'lockdown', 'shielding' and public messaging ('stay at home, protect the NHS, save lives') were initiated in a mass attempt to reduce the effective reproduction number $\left(R_{t}\right)$. The restriction of

'Guy's and St Thomas' NHS Foundation Trust, Department of Oral Surgery, Fl 23, Tower Wing, Great Maze Pond, London, SE1 9RT, UK.

*Correspondence to: Vinod Patel

Email address: vinod.patel@hotmail.co.uk

Refereed Paper.

Accepted 21 May 2020

https://doi.org/10.1038/s41415-020-1716-2 movement of non-essential services forced many working sectors to adjust and adapt their jobs to limit direct personnel contact. However, not all sectors were able to change working patterns, particularly where the jobs involved providing public services. This meant either continuing to provide adapted services, such as the transport sector, or stopping altogether, such as the restaurant and leisure industry.

Dentistry, though a self-standing profession, is widely categorised under the health and social care sector. On 25 March 2020, the Chief Dental Officer (CDO) for England announced that all non-urgent dental care must cease immediately and urgent dental needs requiring clinical input must be managed through referral to a local urgent dental care (UDC) system. ${ }^{6}$ Understandably, constraints had been placed due to the operational environment of dental practice, such as close working proximity to oro-nasal areas involving saliva and blood contaminants, ${ }^{7}$ as well as the creation of aerosol and droplet contact, all considered as potential transmissible mediums for SARS-CoV-2. ${ }^{8,9,10,11,12}$ However, before this guidance, many general dental practitioners (GDPs) and dental departments had independently decided to suspend activity due to the concern of an elevated risk of both contracting and transmitting COVID-19 based on the nature of their work. ${ }^{13}$ A number of clinical procedures and investigations were considered to potentially generate aerosolised contaminated droplets from affected patients. In dentistry, aerosol generating procedures (AGPs) are widespread among routine clinical practice and are associated with most dental procedures that involve the use of mechanical instrumentation, such as dental handpieces, ultrasonic scalers and air-water syringes. ${ }^{7}$ Recent publications, many from countries with high numbers of COVID-19-positive cases and deaths, supported these concerns. ${ }^{14,15}$ As a result of the 'drills down' guidance, routine dental activity stopped, leaving patients with immediate limited access to urgent dental treatment. This was compounded by the delay in establishing local UDC hubs across the country, with the standard operating procedures (SOPs) for these centres only becoming available on 15 April $2020 .^{16}$

Guy's and St Thomas' NHS Foundation Trust (GSTFT) is situated in the borough of Southwark and Lambeth in Central London, considered the epicentre of the UK pandemic. ${ }^{4}$ The Trust has two sites, with the majority of the dental activity 
based at Guy's Hospital in London Bridge while paediatric dentistry is based at St Thomas', adjoined to the Evelina Children's Hospital. The dental school at Guy's has a well-established acute dental care (ADC) department, which operates during weekdays between the hours of $8.30 \mathrm{am}-5$ $\mathrm{pm}$ as a self-referral walk-in for adults only. The service provides care for patients with urgent dental needs who are unable to receive treatment elsewhere. A variety of treatment is provided by the service and patients presenting with more complex pathology requiring further input from other disciplines can be referred onwards for an assessment and management if they fit sub-speciality acceptance criteria.

Considering the hospital was situated in the UK epicentre (Fig. 1), ${ }^{17}$ from 18 March 2020, GSTFT dental directorate changed their SOPs and began to only provide UDC through an adapted and enhanced ADC service. Encompassed by the rapidly developing and ensuing pandemic, the ADC service remained one of the few access points for patients with urgent dental needs following the CDO's decision ${ }^{6}$ to halt all but urgent dental care.

The current article is a service evaluation during a five-week period leading up to the peak ${ }^{18}$ of COVID-19 of a new and adapted system implemented to provide UDC following national cessation of routine dentistry. The article explores and presents the spectrum of diagnoses and treatments that presented to the service within a tertiary hospital based in the UK's epicentre of the COVID-19 pandemic.

\section{Materials and methods}

\section{Patient pathway}

The enhanced ADC service required development of a new protocol to assess and manage urgent care patients based on evolving evidence and guidance from Public Health England (PHE) ${ }^{19}$ and GSTFT COVID-19 safety and tactical team. Additionally, the department contacted the School and Hospital of Stomatology, Wuhan University, ${ }^{20}$ on 21 March 2020, receiving a same-day reply regarding our queries for delivering a service and confirming points in their recently published article. Their recent article described challenges and experiences of providing emergency dental treatment during the COVID-19 outbreak and was taken into consideration for developing our workflow.

The GSTFT ADC service has always been a walk-in, self-referral service and this mode of access was kept open for the local community. Upon attendance and registration, patients

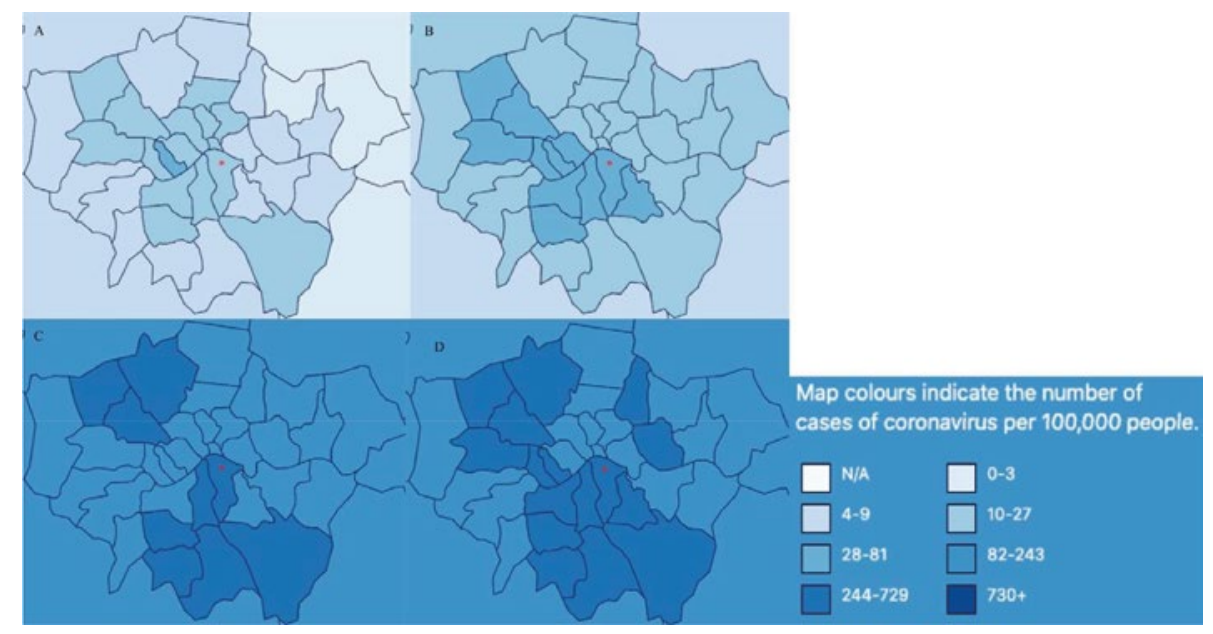

Fig. 1 Shows Southwark borough (red asterisk) in which GSTFT and the enhanced ADC centre is located, highlighting the region to have been a consistent epicentre for COVID-19 during the period of this service evaluation. a) Start of data collection: 18 March 2020. b) First lockdown: 23 March 2020. c) Second lockdown: 16 April 2020. d) End of data collection and peak of COVID-19: 22 April 2020. Figure courtesy of CovidLiveUK ${ }^{17}$

Box 1 Shows the medical condition screening list used to determine vulnerable patients. This list was not exhaustive and clinical judgement was used for additional medical conditions ${ }^{21}$

- Age 70 or older

- Pregnant

- Lung conditions, eg asthma, chronic obstructive pulmonary disease, emphysema or bronchitis

- Heart disease, eg heart failure

- Chronic kidney disease

- Liver disease, eg hepatitis

- Conditions affecting the brain and nerves, eg Parkinson's disease, motor neurone disease, multiple sclerosis, a learning disability or cerebral palsy

- Diabetes

- Conditions affecting the spleen, eg sickle cell disease or spleen removal

- Immunosuppression, eg HIV and AIDS, systemic steroid use or chemotherapy

- BMl of 40 or above

were immediately taken to a triage area where temperatures were taken followed by COVID19-related questions to assess risk of infection and to identify patients within the vulnerable and shielded group (Box 1). ${ }^{21}$ Following normal temperatures and triage negative for COVID-19, patients were asked for the reason for their attendance. If their presenting complaint required urgent treatment, they were subsequently offered a time slot to return and asked to leave the department, maintain social distancing and return at their allotted time.

In addition to this, a telephone triage was set up with NHS England to spread the net of care and to control patient attendance to maintain social distancing in both clinical and non-clinical patient areas. A phone line was dedicated to the ADC service to provide access to patients who had called NHS 111 have spoken to a dental representative who determined they required an urgent appointment. The phone triage followed the same questioning as the walk-in protocol and, if accepted, patients were offered time slots for attendance. Upon arrival, temperatures were taken in the triage area before full clinical assessment. For patients who were unable to attend due to either COVID-19 symptoms or concern regarding shielding, antimicrobials were remotely prescribed when facial swelling or dental infection-related temperature elevation was reported.

Once initial screening had been completed, patients were allocated to have their full clinical assessment at different hubs within the dental hospital to provide appropriate specialist advice 


\begin{tabular}{|c|c|c|c|c|c|c|c|}
\hline & \multirow{2}{*}{$\begin{array}{l}\text { Waiting room/ } \\
\text { reception triage }\end{array}$} & \multicolumn{3}{|c|}{ Triaged COVID-19 negative } & \multicolumn{3}{|c|}{$\begin{array}{l}\text { Triaged COVID-19 } \\
\text { suspected or confirmed positive }\end{array}$} \\
\hline & & $\begin{array}{l}\text { Dental } \\
\text { assessment }\end{array}$ & $\begin{array}{l}\text { Non-AGP } \\
\text { treatment }\end{array}$ & $\begin{array}{l}\text { AGP } \\
\text { treatment }\end{array}$ & $\begin{array}{l}\text { Dental } \\
\text { assessment }\end{array}$ & $\begin{array}{l}\text { Non-AGP } \\
\text { treatment }\end{array}$ & $\begin{array}{l}\text { AGP } \\
\text { treatment }\end{array}$ \\
\hline Good hand hygiene & Yes & Yes & Yes & Yes & Yes & Yes & Yes \\
\hline Disposable gloves & No & Yes & Yes & Yes & Yes & Yes & Yes \\
\hline Fluid-resistant disposable plastic apron & Yes & Yes & No & No & No & No & No \\
\hline Fluid-resistant disposable gown & No & No & Yes & Yes & Yes & Yes & Yes \\
\hline Fluid-resistant surgical mask & Yes & Yes & Yes & Yes & Yes & Yes & Yes \\
\hline Filtering facepiece (FFP3) respirator & No & No & No & Yes & Yes & Yes & Yes \\
\hline Eye protection & Yes & Yes* & Yes* & Yes* & Yes* $/^{* *}$ & Yes* $/^{* *}$ & Yes**** \\
\hline Full face visor shield & No & Yes* & Yes* & Yes* & Yes* & Yes* & Yes* \\
\hline Surgical cap & No & No & No & Yes & Yes & Yes & Yes \\
\hline
\end{tabular}

and maintain distancing. The majority of patients were managed in the ADC area, while patients identified within the 'vulnerable' COVID-19 group were directed to the department of sedation and special care dentistry, situated on a different floor, providing separation from the general public where possible. Paediatric patients were triaged by a separate phone system and seen at the St Thomas' site at the dedicated paediatric dental centre, while some internal orthodontic emergencies were seen at Guy's. During the public holidays, the enhanced Guy's ADC department triaged and managed both paediatric and adult emergencies.

Radiographic investigations were limited to sectional dental panoramic tomographs (DPTs) regarding the area of interest. ${ }^{22}$ Where necessary, additional imaging such as cone beam CT was used in line with the COVID-19 dental radiology guidance. ${ }^{22}$

Following radiographic investigation, patients were given a diagnosis and appropriate treatment options. If teeth required extirpation, this was carried out by the restorative team on a separate floor. Tooth extractions were referred to the department of oral surgery, where a dedicated zone was set up for treatment, which was all provided under local anaesthesia. Patients vulnerable to COVID-19 had all their treatment provided and completed by the sedation and special care team. Almost all treatment was carried out under local anaesthesia, except on rare occasions where intravenous sedation was provided.

Walk-in patients suspected or confirmed positive and symptomatic for COVID-19 were immediately isolated in a dedicated room located

Table 2 Shows the system for classifying emergency dental need ${ }^{26}$

\begin{tabular}{l|l|l} 
Emergency & Urgent & Routine \\
\hline $\begin{array}{l}\text { Trauma including facial/oral laceration } \\
\text { and/or dentoalveolar injuries }\end{array}$ & $\begin{array}{l}\text { Dental and soft-tissue infections } \\
\text { without a systemic effect }\end{array}$ & Mild or moderate pain \\
\hline $\begin{array}{l}\text { Significant and worsening oro-facial } \\
\text { swelling }\end{array}$ & Severe dental and facial pain & Minor dental trauma \\
\hline $\begin{array}{l}\text { Uncontrollable post-extraction bleeding } \\
\text { practured teeth or tooth with } \\
\text { pulpal exposure } \\
\text { comperature as a result of dental }\end{array}$ & $\begin{array}{l}\text { Controllable post-extraction } \\
\text { bleeding }\end{array}$ \\
\hline $\begin{array}{l}\text { Severe trismus } \\
\text { finfection }\end{array}$ & $\begin{array}{l}\text { Fractured, loose or displaced } \\
\text { restorations or prostheses }\end{array}$ \\
\hline $\begin{array}{l}\text { Oro-dental conditions that are likely to } \\
\text { exacerbate systemic medical conditions }\end{array}$ & & $\begin{array}{l}\text { Treatment normally associated } \\
\text { with routine dental care }\end{array}$ \\
\hline
\end{tabular}

close to the triage zone to limit departmental exposure. Patients were provided with a mask and all assessment and treatmeFnt was completed within the COVID-19 room on an expedited pathway to reduce their time within the service.

\section{Personal protective equipment}

Personal protective equipment (PPE) has been a highly debated topic during the COVID-19 pandemic period, evolving with time. GSTFT followed the PHE guidance ${ }^{23,24,25}$ PHE guidance $(23,24,25)$ in combination with the Trust's own SOPs for optimal PPE. Currently, the standards being used are presented in Table 1.

\section{Case severity classification}

Adult walk-in and telephone triage was performed by consultants to ensure that the highest level of clinical judgement and experience was used to determine acceptance for attendance and treatment. For the purpose of categorising treatment, case severity was defined in line with the classification system outlined in the Scottish Dental Clinical Effectiveness Programme (SDCEP) documentation - Emergency Dental Care: Dental Clinical Guidance ${ }^{26}$ (Table 2). Treatments were divided into a binary format: AGPs or non-AGPs, based upon the definitions presented by the Faculty of General Dental Practice (FGDP) ${ }^{27}$ on 20 March 2020, which have subsequently been maintained in a later update. ${ }^{28}$

\section{Data collection}

Data were collected retrospectively from 18 March 2020 until 22 April 2020, spanning the period of the COVID-19 lockdown to the UK's COVID-19 peak. ${ }^{18}$ The former date was following cessation of all elective clinics and 
operating within the dental directorate and the commencement of an enhanced ADC service.

Clinical software systems were used to collect data which were input into secure and encrypted spreadsheet software, which was only accessible by the authors. No identifiable data were collected. Data recorded were broadly split into basic demographic and clinical measures. Demographic measures included the number of attendees, geographical distribution of attendees, age distribution and the proportion of attendees in the 'vulnerable' COVID-19 category (based on criteria listed in Box 1).

Patients' postcodes were noted to capture distances travelled to receive UDC, considering that the standards for commissioning suggest that the usual travel time by public transport for patients seeking urgent care should be no more than one hour. ${ }^{29}$

Clinical information recorded consisted of the diagnosis, including site, management and case severity for each patient. Case severity was determined from criteria described in Table 2. Two independent assessors gauged the clinical severity of each case based on the clinical notes, and where a disagreement arose, a third independent judicator's expert opinion was sought.

\section{Workforce}

Non-identifiable numerical data regarding the workforce which included basic demographic spread and staff sickness numbers were provided by the directorate management and human resources team.

\section{Results}

A total of 1,510 patients were clinically assessed via the enhanced $\mathrm{ADC}$ system, of which 1,107 patients were self-referral walk-ins. The telephone triage process yielded 611 patients, of whom 403 attended ADC for further clinical assessment. The remaining patients were either redirected towards their local secondary care services also providing UDC to reduce travel and exposure time, or managed using the '3A protocol' (advice, analgesia, antimicrobials), ${ }^{6,30}$ particularly for those who were isolating or shielding.

The current cohort had an age range of 5-87 years of age, with an average of 41 (median age 38). An almost equal number of males and females attended with a diverse ethnicity as to be expected for a London population. Only one patient attended via walk-in who was deemed suspected COVID-19-positive and did not require any dental treatment. No confirmed

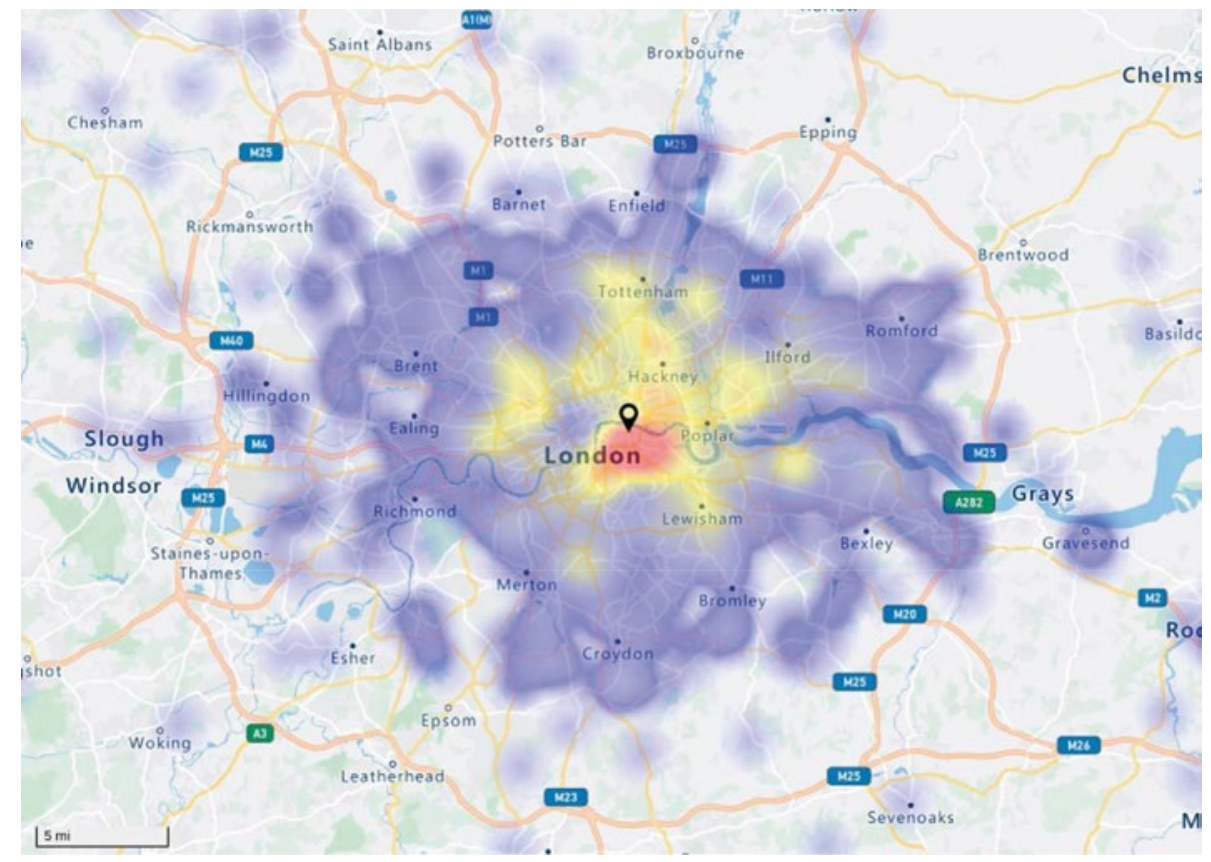

Fig. 2 Shows a heat map of the locations of the 1,510 attendees. The location pin signifies the location of Guy's Hospital. The figure highlights that the hospital has provided a service to the whole of London, as well as the distances patients travelled from outside of London where urgent dental care was limited. Figure courtesy of www.espatial.com

symptomatic COVID-19 patients were seen for face-to-face assessment or treatment. Patients attended from London and beyond as evident in the heat map shown in Figure 2, with the South East London region being the most frequent area of attendance. Patient demographics are outlined in Table 3.

Analysis of the presenting dental diagnoses identified irreversible pulpitis/apical pathology as the most common ( $\mathrm{n}=958,63.4 \%)$. Severity stratifying based on the SDCEP guidance ${ }^{26}$ identified $0.9 \%(\mathrm{n}=14)$ as emergency, $74.2 \%$ $(\mathrm{n}=1,120)$ as urgent and $24.9 \%(\mathrm{n}=376)$ as routine (Fig. 3). A total of $82.1 \%$ of treatments were non-AGPs (extraction 742/1,240, other $498 / 1,240$ ), while the remaining treatments were AGPs (extraction 89/270, extirpation 181/270) (Table 3).

The dental directorate has a total of 879 staff, 486 of whom provide direct clinical care, of which 50.4\% (245/486) are black, Asian and minority ethnic (BAME). The peak age group in the directorate is $35-44$ years $(193 / 486,39.7 \%)$, with over $75 \%(369 / 486)$ of the workforce $<45$ years of age. The Trust's request for staff for redeployment to essential services was fully met by the dental directorate as a matter of priority, with the remaining workforce then assessed for provision of dental services. During the period of this data collection, 231 (full- and part-time) staff members (55 dental consultants, 78 non-consultant-grade clinicians and 102 nurses/allied health professionals/patient administration) provided patient-facing dental services at some point during the five-week service evaluation. The total number did not discriminate against the number of days worked and therefore included those that had provided only a single day, multiple days or every day of the five-week period. The remaining clinical staff were either based at St Thomas' Paediatric Dental Centre, redeployed to essential services, were shielding or provided solely non-patient-facing care such as telephone or video consultations. A small group of dentists provided daily inpatient oral care with a focus to avoid ventilator- and non-ventilator-related pneumonia. Of the 133 clinicians, 23 provided all the AGPs across the various sub-specialities on a rota-based system, with no clinician required to provide AGPs on consecutive days.

\section{Discussion}

The current COVID-19 pandemic has led to dramatic and instant change for the delivery of dental care. Understandably, routine and elective dental treatment has stopped; however, UDC has been encouraged with the use of appropriate PPE. Considering both NHS and private primary care has been nationally paralysed, a vacuum was initially present for the management of urgent 
cases. Though some secondary care services such as dental hospitals had continued to provide dental emergency services, they had limited capacity due to depletion in workforce, either from redeployment to other essential services or due to COVID-19 illness. GDPs continued to provide a non-contact emergency service with clear limitations, which highlighted the integral service the primary care practitioner provides, incorporating UDC into their daily routine care by accommodating their diaries. This approach is often taken for granted and overlooked and is most obvious now that dentistry has been essentially halted, leaving a substantial patient population with UDC needs. This is demonstrated by the London NHS 111 call figures, where recent feedback reported $80 \%$ of all non-COVID-19 calls were dentally related. For the period of 1 April 2020 to 23 April 2020, a total of 17,069 calls were received by the dental nurse triage section of London NHS 111. The ADC phone triage received an average of 24 calls per three-hour session and over the service evaluation period took 611 calls.

\section{Management of dental emergencies}

With the limitation placed on dental care, many have looked for guidance towards managing dental emergencies. The CDO has proposed first-line treatment using telephone triage and the ' $3 \mathrm{~A}$ approach.',30 Beyond this, escalation of the patients to an UDC pathway ${ }^{16}$ may be required. In addition to this, the 2007 document Emergency Dental Care: Dental Clinical Guidance ${ }^{26}$ has commonly been shared and cited by dentists so they may be able to help their patients. The document estimates that approximately $1 \%, 75 \%$ and $25 \%$ of 'emergency' calls will fall into the respective categories of emergency, urgent and routine clinical need, and our figures of $0.9 \%$, $74.2 \%$ and $24.9 \%$ reflect this. The document stratifies patients in an assumed normalised time in which routine dental services are available. In the pandemic situation, conditions described as 'routine' may need escalating. For instance, under the routine category is mild to moderate orofacial pain, which in 'normal times' a dentist may advise analgesia as an interim measure until an appointment can be made to provide a more definitive solution. With no proposed national or dental exit strategy, ${ }^{31}$ the likelihood is progressive and prolonged suffering and increasing severity of symptoms for patients. A recent piece by the President of the British Association of Oral Surgeons highlighted a salient point that 'early management of acute dental emergencies is important to prevent patients from attending
Accident and Emergency services and to avoid hospital admissions. One concern is that with the suspension of routine dental care, more patients than usual could need admission for the management of acute dental infections that threaten the airway and require intensive care. ${ }^{32}$ Considering the national service pressures on intensive care, inpatient beds and reduction of urgent general anaesthesia availability due to COVID-19, it is prudent to manage these cases

Table 3 Shows a breakdown of patient demographics and outcomes for the 1,510 enhanced ADC service attendees

\begin{tabular}{|c|c|c|c|}
\hline & Category & Sub-category & $\begin{array}{l}\text { Number } \\
\text { (percentage) }\end{array}$ \\
\hline \multirow{11}{*}{$\begin{array}{l}\text { Patient } \\
\text { demographics }\end{array}$} & \multirow{3}{*}{ Age } & Range & $5-87$ \\
\hline & & Average & 41 \\
\hline & & Median & 38 \\
\hline & \multirow{2}{*}{ Gender } & Male & $774(51.3 \%)$ \\
\hline & & Female & $736(48.7 \%)$ \\
\hline & \multirow{6}{*}{ Ethnicity } & White & $401(26.6 \%)$ \\
\hline & & Mixed & $58(3.8 \%)$ \\
\hline & & Asian or Asian British & $77(5.1 \%)$ \\
\hline & & Black or black British & $154(10.2 \%)$ \\
\hline & & Other & $36(2.4 \%)$ \\
\hline & & Not specified & $784(51.9 \%)$ \\
\hline \multirow{5}{*}{ Diagnoses } & & Irreversible pulpal/apical pathology & $958(63.4 \%)$ \\
\hline & & Reversible pulpal pathology & $22(1.5 \%)$ \\
\hline & & Odontogenic infection & $134(8.9 \%)$ \\
\hline & & Trauma & $103(6.8 \%)$ \\
\hline & & Other & $293(19.4 \%)$ \\
\hline \multirow{8}{*}{ Management } & \multirow{3}{*}{ AGPs } & Surgical extraction & $89(5.9 \%)$ \\
\hline & & Extirpation & $181(12 \%)$ \\
\hline & & AGP total & $270(17.9 \%)$ \\
\hline & \multirow{5}{*}{ Non-AGPs } & Simple extraction & $742(49.1 \%)$ \\
\hline & & Prescription only & $142(9.4 \%)$ \\
\hline & & Advice only & $187(12.4 \%)$ \\
\hline & & Other & $169(11.2 \%)$ \\
\hline & & Non-AGP total & $1,240(82.1 \%)$ \\
\hline \multirow{11}{*}{ Geography } & \multirow{9}{*}{ Inner London } & North West London & $98(6.5 \%)$ \\
\hline & & North London & $150(9.9 \%)$ \\
\hline & & East London & $249(16.5 \%)$ \\
\hline & & East Central London & $11(0.7 \%)$ \\
\hline & & South East London & $317(21.0 \%)$ \\
\hline & & South West London & $135(8.9 \%)$ \\
\hline & & Western Central London & $8(0.5 \%)$ \\
\hline & & West London & $81(5.4 \%)$ \\
\hline & & Inner London total & $1,049(69.5 \%)$ \\
\hline & \multicolumn{2}{|c|}{ Greater London } & $406(26.9 \%)$ \\
\hline & \multicolumn{2}{|c|}{ Outside of London } & $55(3.6 \%)$ \\
\hline
\end{tabular}


Fig. 3 Shows the number and severity grading of attendees and their diagnoses based on the SDCEP classification ${ }^{26}$

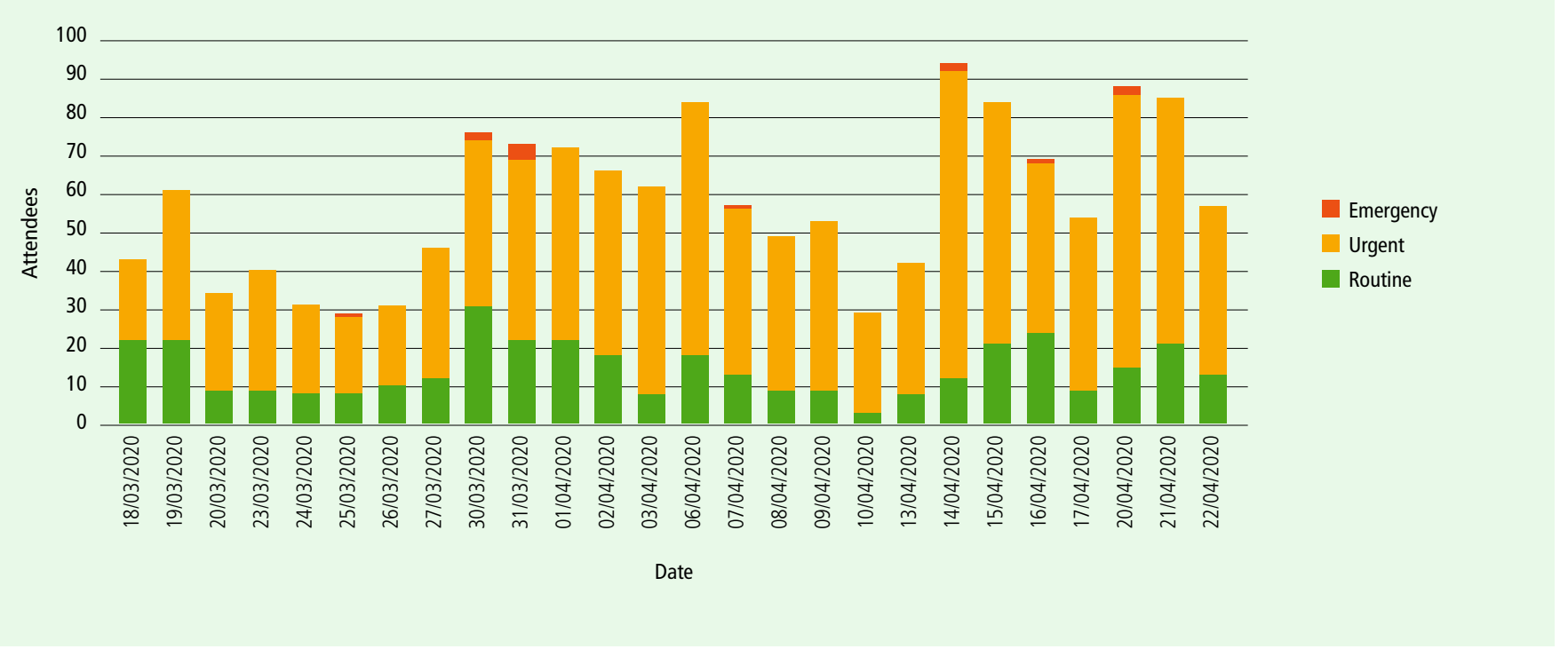

expediently with effective treatments such as extractions or extirpations, as opposed to the 3 As, which does not reliably alleviate symptoms for what is commonly considered as 'toothache' (63.4\%). Based on our frontline experience, many of the patients seen had already received the 3 As, some with multiple courses of antimicrobials, and many of these prescriptions were not in line with the appropriate indication ${ }^{33}$ of use. The latter was actioned by dentists understandably out of desperation as no other intervention was possible and there was a lack of local UDC provisions available. The FGDP rightly recognised the limitations of the $3 \mathrm{As}^{34}$ and that, although 50 UDC centres were opened, a significant gap in capacity still existed..$^{28}$ Their statement is supported by the geographical distribution of the origin of the patients in the current service evaluation, with almost $80 \%$ from outside our local area, most probably explained by the significant delays in UDC setups across home counties due to the complexities involved. Taking these points into consideration, although the SDCEP criteria ${ }^{26}$ suggests that $24.9 \%$ of our cases were routine, in the authors' opinion, these should be reclassified as urgent. Reclassifying the mild to moderate pain group meant only $116 / 1,510(7.7 \%)$ of our overall cohort can be retrospectively considered routine.

\section{Patient demographics}

It has been well publicised that certain patient groups have a heightened risk of morbidity and mortality from COVID-19. ${ }^{21}$ The government therefore requested during the lockdown that vulnerable patients should consider shielding for 12 weeks as a protective approach and to reduce their risk. The enhanced ADC service managed a substantial number of patients ( $\mathrm{n}=338)$ under this heading, which highlights the urgency and importance that these patients had placed on their dental problem to break their shielding. The $3 \mathrm{~A}$ approach for pulpitic teeth in this group on the background of a 12 -week shielding phase is an unsatisfactory intervention with the potential for deterioration, and the impact on their medical conditions remains a viable justification to intervene to provide UDC. Assessing our vulnerable group, the enhanced ADC system saw 338 patients and, based on our own severity stratification, $303 / 338$ (89.6\%) were urgent cases while 2/338 $(0.6 \%)$ were emergency.

\section{Workforce}

Dentistry at GSTFT is its own directorate; however, the various sub-specialities and dedicated location within the Trust allows it to informally act as a hospital within a hospital. The advantage of this is the availability of a large workforce to compensate for sickness and redeployment while still maintaining a service.

Following the evolving COVID-19 advice from the government until 22 April 2020, a total of 133/879 staff had registered as not being able to attend work in relation to coronavirus, either due to direct symptoms or indirectly due to household symptoms. Understandably, there is angst within the dental community about the occupational hazard of COVID-19 and this should be taken seriously. Considering this concern, a five-week service evaluation of an enhanced ADC service provides an interesting window for analysis of the effectiveness of PPE for frontline dentistry. COVID-19 can silently incubate for up to 14 days before symptoms appear. ${ }^{35}$ Hence, discounting the first two weeks of the five-week enhanced ADC service excludes staff who may have contracted COVID-19 before the SOPs were in place. Analysis of three weeks of clinical service (1 April 2020-22 April 2020) leading to the UK COVID-19 peak $^{18}$ and the potential for 14 days of silent incubation before symptoms (6 May 2020 ) identified $2 / 231$ patient-facing staff who reported COVID-19 symptoms and required isolation. In both cases, the individuals were not operating clinicians and symptoms were mild with no hospital admission required. Though both staff members had symptoms consistent with COVID-19, this had not been objectively determined by testing at the time of writing. The authors recognise that $80 \%{ }^{36}$ of individuals with COVID-19 can be either asymptomatic or have only mild symptoms; however, without both mass and reliable testing, exact numbers of COVID-19 positivity are indeterminate at this stage. Testing and the criteria surrounding it are constantly evolving. As we are now able to rapidly test, staff experiencing symptoms are now seen immediately, which allows for isolation or return to work with an element of confidence. This lessens the need to erode the workforce before testing. Considering the Imperial model estimates $2.7 \%(1.2-5.4 \%)$ of the UK population to be infected with COVID$19,{ }^{37}$ their figures would suggest that, in our workforce population, 1.2 (0.5-2.3) individuals would be COVID-19-positive regardless of their occupation and, therefore, based on the limited information, our staff did not show an elevated 
symptom rate. In comparison, the Wuhan University School and Hospital of Stomatology reported no infections in their 169 emergency staff treating over 700 patients, ${ }^{20}$ while in contrast, GSTFT treated over 1,500 patients with 231 staff.

\section{Conclusions}

There is a clear need for UDC, and provisions should be made even during a pandemic. Although a phone triage system providing advice, analgesia and antimicrobials can be easily instigated, it has significant limitations, particularly in diagnoses involving a necrotic pulp where the evidence-based treatment options including extraction or root canal therapy to achieve symptomatic relief should be used. There is no evidence of increased transmission of COVID-19 to frontline dental staff over that expected in the general population if appropriate PPE is correctly used. It would be valuable to determine whether other dental emergency departments and primary care UDC hubs have had similar experiences.

The current service evaluation is comparable to that of the School and Hospital of Stomatology, Wuhan University, based on the volume of patient contact and being operational within a pandemic epicentre.

\section{Acknowledgements}

The authors would like to thank all the clinical and non-clinical staff within the dental directorate at GSTFT who were involved in the enhanced $A D C$ system. The teamwork between all dental sub-specialities, management and administrative staff enabled a rapid and adaptive system to be implemented and allowed for provision of urgent and emergency dental services. Specifically, we would like to thank and acknowledge the help and assistance of Dr Mona Hajatdoost-Sani. The authors would like to acknowledge and thank Joanna Johnson and Eric Whaites (Joint Clinical Directors of the dental directorate, GSTFT) for their support in undertaking this project and in preparation of this manuscript. Further acknowledgement and thanks to the London region of NHS England and NHS Improvement for their input and assistance working with the dental directorate at GSTFT in developing urgent dental care provisions. We would also like to thank Dr Z. Bian (School and Hospital of Stomatology, Wuhan University) for kindly responding to our queries.

Author contributions

S. Grossman and P. Sandhu are joint first authors.

Conflict of interest

There are no conflicts of interest to declare by any of the authors for this submitted work.

\section{References}

1. Li Q, Guan X, Wu P et al. Early Transmission Dynamics in Wuhan, China, of Novel Coronavirus-Infected Pneumonia. N Engl J Med 2020; 382: 1199-1207.

2. WHO. Director-General's opening remarks at the media briefing on COVID-19 - 11 March 2020. 2020. Available at https://www.who.int/dg/speeches/detail/who-directorgeneral-s-opening-remarks-at-the-media-briefing-oncovid-19---11-march-2020 (accessed June 2020).

3. Roser M, Ritchie H, Ortiz-Ospina E, Hasell J. Coronavirus Pandemic (COVID-19). 2020. Available at https:// ourworldindata.org/coronavirus (accessed May 2020).

4. UK Government. Coronavirus (COVID-19) in the UK. 2020. Available at https://coronavirus.data.gov.uk/ (accessed May 2020)

5. UK Government. PM address to the nation on coronavirus: 23 March 2020. 2020. Available at https://www.gov.uk/government/speeches/ pmaddresstothenationoncoronavirus 23 march-2020 (accessed May 2020).

6. Hurley S, Neligan M. Issue 3: Preparedness letter for primary dental care - 25 March 2020. 2020. Available at https://www.england.nhs.uk/coronavirus/wp-content/ uploads/sites/52/2020/03/issue-3-preparedness-letterfor-primary-dental-care-25-march-2020.pdf (accessed April 2020).

7. Harrel S K, Molinari J. Aerosols and splatter in dentistry. J Am Dent Assoc 2004; 135: 429-437.

8. Liu J, Liao X, Qian S et al. Community Transmission of Severe Acute Respiratory Syndrome Coronavirus 2. Shenzhen, China, 2020. Emerg Infect Dis 2020; 26: 1320-1323.

9. Chan J, Yuan S, Kok Ket al. A familial cluster of pneumonia associated with the 2019 novel coronavirus indicating persontoperson transmission: a study of a family cluster. Lancet 2020; 395: 514-523.

10. Li Q, Guan X, Wu P et al. Early Transmission Dynamics in Wuhan, China, of Novel Coronavirus-Infected Pneumonia. N Engl J Med 2020; 382: 1199-1207.

11. Huang C, Wang Y, Li X et al. Clinical features of patients infected with 2019 novel coronavirus in Wuhan, China. Lancet 2020; 395: 497-506.

12. Burke R, Midgley C, Dratch A et al. Active Monitoring of Persons Exposed to Patients with Confirmed COVID-19 - United States, January-February 2020. MMWR Morb Mortal Wkly Rep 2020; 69: 245-246.

13. Gamio L. The Workers Who Face the Greatest Coronavirus Risk. The New York Times (New York) 2020 March 15.

14. Peng $X, X u X, L i$, Cheng L, Zhou X, Ren B. Transmission routes of 2019-nCoV and controls in dental practice. Int J Oral Sci 2020: 12: 9.

15. Izzetti R, Nisi M, Gabriele M, Graziani F. COVID-19 Transmission in Dental Practice: Brief Review of Preventive Measures in Italy. J Dent Res 2020; DOI: 10.1177/0022034520920580

16. NHS England and NHS Improvement. COVID-19 guidance and standard operating procedure. 2020. Available at https://www.england.nhs.uk/coronavirus/wp-content/ uploads/sites/52/2020/06/C0581-covid-19-urgent-dentalcare-sop-update-16-june-20-.pdf (accessed May 2020).

17. CovidLiveUK. Live coronavirus figures. 2020. Available online at https://www.covidlive.co.uk/ (accessed May 2020).

18. UK Parliament. Health Secretary: "We are at the peak". 2020. Available at https://www.parliament.uk/business/ news/2020/april1/covid-19-statement-22-april/ (accessed May 2020).

19. UK Government. COVID-19: guidance for health professionals. 2020. Available at https://www.gov. uk/government/collections/wuhan-novel-coronavirus (accessed May 2020).

20. Meng L, Hua F, Bian Z. Coronavirus Disease 2019 (COVID 19): Emerging and Future Challenges for Dental and Oral Medicine. J Dent Res 2020; 99: 481-487.

21. UK Government. Staying at home and away from others (social distancing). 2020. Available online at https://www. gov.uk/government/publications/fullguidanceonstayingat homeandawayfrom-others/fullguidanceonstayingathomea ndawayfrom-others (accessed May 2020)

22. Royal College of Surgeons of England. Recommendations for Diagnostic Imaging during COVID-19 pandemic. 2020 Available online at https://www.rcseng.ac.uk/-/media/ files/rcs/fds/guidelines/dentalradiographycovid19.pdf (accessed May 2020).

23. Public Health England. Recommended PPE for healthcare workers by secondary care inpatient clinical setting, NHS and independent sector. 2020. Available at https://assets.publishing.service.gov.uk/government/ uploads/system/uploads/attachment_data/file/879107/ T1_poster_Recommended_PPE_for_healthcare_ workers_by_secondary_care_clinical_context.pdf (accessed May 2020).

24. Public Health England. Recommended PPE for primary, outpatient, community and social care by setting, NHS and independent sector. 2020. Available at https:// assets.publishing.service.gov.uk/government/uploads/ system/uploads/attachment_data/file/878750/ T2_poster_Recommended_PPE_for_primary outpatient__community_and_social_care_by_setting. pdf (accessed May 2020).

25. Public Health England. Additional considerations, in addition to standard infection prevention and control precautions, where there is sustained transmission of COVID-19, taking into account individual risk assessment for this new and emerging pathogen, NHS and independent sector. 2020. Available at https://assets. publishing.service.gov.uk/government/uploads/system/ uploads/attachment_data/file/879111/T4_poster Recommended_PPE_additional_considerations_of COVID-19.pdf (accessed May 2020).

26. Scottish Dental Clinical Effectiveness Programme. Emergency Dental Care: Dental Clinical Guidance. 2007. Available at https://www.sdcep.org.uk/wp-content/ uploads/2013/03/EDC+Guidance.pdf (accessed May 2020).

27. Faculty of General Dental Practice. COVID-19: new guidance and support for the 'delay' phase. 2020. Available at https://www.fgdp.org.uk/news/covid-19-new-guidanceand-support- $\%$ E2\%80\%98delay $\%$ E2\%80\%99-phase (accessed May 2020).

28. Faculty of General Dental Practice. COVID-19: latest guidance and resources for GDPs. 2020. Available at https://www.fgdp.org.uk/news/ covid19latestguidanceandresourcesgdps-0 (accessed May 2020).

29. NHS England and NHS Improvement. Commissioning Standard for Urgent Dental Care. 2019. Available at https://www.england.nhs.uk/wp-content/ uploads/2019/07/commissioning-standard-for-urgentdental-care.pdf (accessed May 2020).

30. NHS England and NHS Improvement. Dental services webinar: 3 April 2020. 2020. Available online at https:// youtu.be/pkQk3lw0oJU (accessed May 2020).

31. Royal College of Surgeons of England. COVID-19 Q\&A with the Deans of the Dental Faculties. 2020. Available at https://www.rcseng.ac.uk/newsandevents/events/ calendar/webinarcovid19fds7april2020/ (accessed May 2020).

32. Dave M, Seoudi N, Coulthard P. Urgent dental care for patients during the COVID-19 pandemic. Lancet 2020; 395: 1257.

33. Scottish Dental Clinical Effectiveness Programme. Drug Prescribing for Dentistry: Dental Clinical Guidance. 2016. Available at https://www.sdcep.org.uk/wp-content/ uploads/2016/03/SDCEP-Drug-Prescribing-for-Dentistry3rd-edition.pdf (accessed May 2020).

34. Faculty of General Dental Practice. Open letter on prescribing antibiotics during COVID-19. 2020. Available at https://www.fgdp.org.uk/news/openletterprescribingan tibioticsduringcovid19-\%C2\%A0 (accessed May 2020).

35. WHO. Q\&A on coronaviruses (COVID-19). 2020. Available online at https://www.who.int/emergencies/diseases/ novel-coronavirus-2019/question-and-answers-hub/q-adetail/q-a-coronaviruses (accessed June 2020)

36. WHO. Coronavirus disease 2019 (COVID-19) Situation Report - 46. 2020. Available at https://www.who. int/docs/default-source/coronaviruse/situationreports/20200306-sitrep-46-covid-19.pdf (accessed May 2020).

37. Flaxman S, Mishra S, Gandy A et al. Report 13: Estimating the number of infections and the impact of nonpharmaceutical interventions on COVID-19 in 11 European countries. 2020. Available at https://www.imperial. ac.uk/media/imperial-college/medicine/sph/ide/gidafellowships/Imperial-College-COVID19-Europe-estimates and-NPI-impact-30-03-2020.pdf (accessed May 2020). 